\title{
On the computation of the determinant of vector-valued Siegel modular forms
}

\author{
Sho Takemori
}

\section{ABSTRACT}

Let $A^{0}\left(\Gamma_{2}\right)$ denote the ring of scalar-valued Siegel modular forms of degree two, level 1 and even weights. In this paper, we prove the determinant of a basis of the module of vector-valued Siegel modular forms $\bigoplus_{k \equiv \epsilon \bmod 2} A_{\operatorname{det}^{k} \otimes \operatorname{Sym}(j)}\left(\Gamma_{2}\right)$ over $A^{0}\left(\Gamma_{2}\right)$ is equal to a power of the cusp form of degree two and weight 35 up to a constant. Here $j=4,6$ and $\epsilon=0,1$. The main result in this paper was conjectured by Ibukiyama (Comment. Math. Univ. St. Pauli 61 (2012) 51-75).

\section{Introduction}

Let $\operatorname{Sym}(j)$ be the symmetric tensor representation of $\mathrm{GL}_{2}(\mathbb{C})$ of degree $j$ and $\Gamma_{2}$ the full Siegel modular group of degree two. We denote by $A_{k, j}\left(\Gamma_{2}\right)$ the space of vector-valued Siegel modular forms of degree two, level 1 and weight $\operatorname{det}^{k} \otimes \operatorname{Sym}(j)$. When $j=0$, we simply denote $A_{k}\left(\Gamma_{2}\right)=A_{k, 0}\left(\Gamma_{2}\right)$. We put

$$
A_{\mathrm{Sym}(j)}^{0}\left(\Gamma_{2}\right)=\bigoplus_{k \equiv 0 \bmod 2} A_{k, j}\left(\Gamma_{2}\right), \quad A_{\mathrm{Sym}(j)}^{1}\left(\Gamma_{2}\right)=\bigoplus_{k \equiv 1 \bmod 2} A_{k, j}\left(\Gamma_{2}\right) .
$$

When $j=0$, we denote $A^{0}\left(\Gamma_{2}\right)=A_{\operatorname{Sym}(0)}^{0}\left(\Gamma_{2}\right)$. Then $A_{\operatorname{Sym}(j)}^{0}\left(\Gamma_{2}\right)$ and $A_{\operatorname{Sym}(j)}^{1}\left(\Gamma_{2}\right)$ become $A^{0}\left(\Gamma_{2}\right)$ modules. As is well known, Igusa [8] gave explicit generators and the structure of the ring of Siegel modular forms of degree two:

$$
A^{0}\left(\Gamma_{2}\right)=\mathbb{C}\left[\phi_{4}, \phi_{6}, \chi_{10}, \chi_{12}\right] ;
$$

the modular forms $\phi_{4}, \phi_{6}, \chi_{10}, \chi_{12}$ are algebraically independent over $\mathbb{C}$;

$$
\bigoplus_{k} A_{k}\left(\Gamma_{2}\right)=A^{0}\left(\Gamma_{2}\right) \oplus \chi_{35} A^{0}\left(\Gamma_{2}\right) \text {. }
$$

Here $\phi_{k}$ is the Siegel-Eisenstein series of weight $k$ (for $k=4,6$ ) and $\chi_{k}$ is a cusp form weight $k$ (for $k=10,12,35$ ). It is known that there exists a unique irreducible polynomial $P$ of four variables which satisfies $\chi_{35}^{2}=\chi_{10} P\left(\phi_{4}, \phi_{6}, \chi_{10}, \chi_{12}\right)$. Thus we also have

$$
\bigoplus_{k} A_{k}\left(\Gamma_{2}\right) \cong \mathbb{C}\left[t_{4}, t_{6}, t_{10}, t_{12}, t_{35}\right] /\left(t_{35}^{2}-t_{10} P\left(t_{4}, t_{6}, t_{10}, t_{12}\right)\right),
$$

where $t_{i}(i=4,6,10,12,35)$ are variables.

The generators of the modules $A_{\mathrm{Sym}(j)}^{0}\left(\Gamma_{2}\right)$ and $A_{\mathrm{Sym}(j)}^{1}\left(\Gamma_{2}\right)$ and the fundamental relations among them have been examined by several authors. Satoh [11] proved the structure theorem for $A_{\mathrm{Sym}(2)}^{0}\left(\Gamma_{2}\right)$. To prove the linear independence of some functions over $A^{0}\left(\Gamma_{2}\right)$, he showed

Received 27 February 2014; revised 23 May 2014.

2010 Mathematics Subject Classification 11F46 (primary), 11F03, 11F11, 11F60 (secondary).

Contributed to the Algorithmic Number Theory Symposium XI, GyeongJu, Korea, 6-11 August 2014. 
the nonvanishing of the following generalized Wronskian:

$$
\operatorname{det}\left(\begin{array}{cccc}
4 \phi_{4} & 6 \phi_{6} & 10 \chi_{10} & 12 \chi_{12} \\
\partial_{1} \phi_{4} & \partial_{1} \phi_{6} & \partial_{1} \chi_{10} & \partial_{1} \chi_{12} \\
\partial_{2} \phi_{4} & \partial_{2} \phi_{6} & \partial_{2} \chi_{10} & \partial_{2} \chi_{12} \\
\partial_{3} \phi_{4} & \partial_{3} \phi_{6} & \partial_{3} \chi_{10} & \partial_{3} \chi_{12}
\end{array}\right) .
$$

Here $Z=\left(\begin{array}{ll}z_{11} & z_{12} \\ z_{12} & z_{22}\end{array}\right)$ is the parameter of the Siegel upper half space $\mathbb{H}_{2}$ of degree two and

$$
\partial_{1}=\frac{\partial}{\partial z_{11}}, \quad \partial_{2}=\frac{\partial}{\partial z_{12}}, \quad \partial_{3}=\frac{\partial}{\partial z_{22}} .
$$

Aoki and Ibukiyama [1] proved the Wronskian (1.1) is a non-zero constant multiple of $\chi_{35}$.

The Wronskian (1.1) can be interpreted as follows. Let $\bigwedge^{j+1} \operatorname{Sym}(j)$ be the $j+1$ th exterior product of $\operatorname{Sym}(j)$. Then as representations of $\mathrm{GL}_{2}(\mathbb{C})$, we have $\bigwedge^{j+1} \operatorname{Sym}(j) \cong \operatorname{det}^{1 / 2 j(j+1)}$. Take $j+1$ non-negative integers $k_{1}, \ldots, k_{j+1}$ and $j+1$ vector-valued Siegel modular forms $f_{i} \in$ $A_{k_{i}, j}\left(\Gamma_{2}\right)$ for $1 \leqslant i \leqslant j+1$. We $\operatorname{define} \operatorname{det}\left(f_{1}, \ldots, f_{j+1}\right)$ by $f_{1} \wedge \ldots \wedge f_{j+1}$. Then $\operatorname{det}\left(f_{1}, \ldots, f_{j+1}\right)$ is a vector-valued Siegel modular of weight $\operatorname{det}^{k_{1}+\ldots+k_{j+1}} \otimes \bigwedge^{j+1} \operatorname{Sym}(j)$. We fix a basis of the one-dimensional space $\bigwedge^{j+1} \operatorname{Sym}(j)$ and consider $\operatorname{det}\left(f_{1}, \ldots, f_{j+1}\right)$ as an element of $A_{k}\left(\Gamma_{2}\right)$, where $k=\sum_{i=1}^{j+1} k_{i}+1 / 2 j(j+1)$. Let $f_{1}=\left[\phi_{4}, \phi_{6}\right], f_{2}=\left[\phi_{4}, \chi_{10}\right]$ and $f_{3}=\left[\phi_{4}, \chi_{12}\right]$ be the first three generators of $A_{\mathrm{Sym}(2)}^{0}\left(\Gamma_{2}\right)$ given by Satoh [11]. Then, it is easy to see that (1.1) is a non-zero constant multiple of $\phi_{4}^{-2} \operatorname{det}\left(f_{1}, f_{2}, f_{3}\right)$. Thus we see that $\operatorname{det}\left(f_{1}, f_{2}, f_{3}\right)$ is a non-zero constant multiple of $\phi_{4}^{2} \chi_{35}$.

For $V=A_{\mathrm{Sym}(j)}^{0}\left(\Gamma_{2}\right)$ (respectively $\left.A_{\mathrm{Sym}(j)}^{1}\left(\Gamma_{2}\right)\right)$, we put $j(V)=j$ and $\epsilon(V)=0$ (respectively 1 ). Define sets $\mathfrak{S}$ and $\mathfrak{T}$ by

$$
\begin{aligned}
& \mathfrak{S}=\left\{A_{\mathrm{Sym}(4)}^{0}\left(\Gamma_{2}\right), A_{\mathrm{Sym}(4)}^{1}\left(\Gamma_{2}\right), A_{\mathrm{Sym}(6)}^{0}\left(\Gamma_{2}\right)\right\}, \\
& \mathfrak{T}=\left\{A_{\operatorname{Sym}(6)}^{1}\left(\Gamma_{2}\right), A_{\mathrm{Sym}(8)}^{0}\left(\Gamma_{2}\right), A_{\mathrm{Sym}(8)}^{1}\left(\Gamma_{2}\right)\right\} .
\end{aligned}
$$

In [7], Ibukiyama gave generators and fundamental relations for $V=A_{\operatorname{Sym}(2)}^{1}\left(\Gamma_{2}\right)$ and $V \in \mathfrak{S}$. He proved $V$ is a free $A^{0}\left(\Gamma_{2}\right)$ module of rank $j(V)+1$ for $V \in \mathfrak{S}$ (in $\S 3$, we shall prove that if $A_{\operatorname{Sym}(j)}^{\epsilon}\left(\Gamma_{2}\right)$ is a free $A^{0}\left(\Gamma_{2}\right)$ module then its rank is equal to $\left.j+1\right)$. It is proved that $V$ is also free of rank $j(V)+1$ for $V \in \mathfrak{T}$. This work was done by van Dorp [14] for $V=A_{\operatorname{Sym}(6)}^{1}\left(\Gamma_{2}\right)$ and by Kiyuna [9] for $V=A_{\mathrm{Sym}(8)}^{0}\left(\Gamma_{2}\right)$ and $A_{\mathrm{Sym}(8)}^{1}\left(\Gamma_{2}\right)$. To prove the linear independence of generators, Ibukiyama proved the nonvanishing of the determinant of generators of $V \in \mathfrak{S}$. By the calculation of weights and Fourier-Jacobi expansion, he conjectured the following statement.

Conjecture 1.1 (Ibukiyama). For $V \in \mathfrak{S} \cup \mathfrak{T}$, let $f_{1}, \ldots, f_{j+1}$ be the basis of $V$ over $A^{0}\left(\Gamma_{2}\right)$. Then the determinant $\operatorname{det}\left(f_{1}, \ldots, f_{j+1}\right)$ is a non-zero constant multiple of $\chi_{35}^{j(V) / 2+\epsilon(V)}$.

In this paper, we prove the following statement.

Theorem 1.2. With the notation above, the determinant $\operatorname{det}\left(f_{1}, \ldots, f_{j+1}\right)$ is a non-zero constant multiple of $\chi_{35}^{j(V) / 2+\epsilon(V)}$ if $(j(V), \epsilon(V))=(4,0),(4,1),(6,0)$ or $(6,1)$.

To prove the main result, we use Sage [12] and a Sage package for Siegel modular forms of degree two written by the author. The package can be found at https://github.com/stakemori/ degree2.

C. Citro, A. Ghitza, N.-P. Skoruppa, M. Raum, N. Ryan and G. Tornarìa also wrote a Sage package for Siegel modular forms of degree two (see http://trac.sagemath.org/ticket/8701). 
They implemented a function that computes the multiplication of two Fourier expansions of Siegel modular forms. But it seems that they did not implement a function that computes the multiplication of two Fourier expansions that are not necessarily Siegel modular forms. In this paper, we have to compute many Rankin-Cohen type differential operators. And this computation needs a function that computes the multiplication of derivatives of Siegel modular forms. Since it is not safe to modify such a low-level function as computes the multiplication of Fourier expansions, the author wrote his own package.

\section{Definition}

We review the definition of vector-valued Siegel modular forms of degree two. Define the symplectic group of degree two by

$$
\mathrm{Sp}_{2}(\mathbb{R})=\left\{g \in \mathrm{GL}_{4}(\mathbb{R}) \mid{ }^{t} g w_{2} g=w_{2}\right\}
$$

where $w_{2}=\left(\begin{array}{cc}0_{2} & -1_{2} \\ 1_{2} & 0_{2}\end{array}\right)$. Put $\Gamma_{2}=\operatorname{Sp}_{2}(\mathbb{R}) \cap \mathrm{GL}_{4}(\mathbb{Z})$. We denote by $\left(\operatorname{Sym}(j), V_{j}\right)$ the symmetric tensor representation of $\mathrm{GL}_{2}(\mathbb{C})$. We identify $V_{j}$ with the space of homogeneous polynomials $P\left(u_{1}, u_{2}\right)$ in $u_{1}, u_{2}$ of degree $j$. The action of $g \in \mathrm{GL}_{2}(\mathbb{C})$ is given by $(\operatorname{Sym}(j)(g) P)(u)=P(u g)$ where $u=\left(u_{1}, u_{2}\right)$. For a $V_{j}$-valued function $F$ on the Siegel upper half space $\mathbb{H}_{2}$ of degree two, non-negative integer $k$ and $g=\left(\begin{array}{ll}a & b \\ c & d\end{array}\right) \in \mathrm{GL}_{2}(\mathbb{C})$, we put

$$
\left(\left.F\right|_{k, j}[g]\right)(Z)=\operatorname{det}(c Z+d)^{-k} \operatorname{Sym}(j)(c Z+d)^{-1} F(g Z) \quad \text { for } Z \in \mathbb{H}_{2} .
$$

A $V_{j}$-valued holomorphic function $F$ on $\mathbb{H}_{2}$ is said to be a vector-valued Siegel modular form of weight $\operatorname{det}^{k} \otimes \operatorname{Sym}(j)$ if and only if $\left.F\right|_{k, j}[\gamma]=F$ for all $\gamma \in \Gamma_{2}$. We call $k$ the determinant weight of $F$. It is easy to see that $A_{k, j}\left(\Gamma_{2}\right)=0$ if $j$ is odd.

\section{Hilbert-Poincaré series}

In this section, we prove that the rank of $A_{\mathrm{Sym}(j)}^{\epsilon}\left(\Gamma_{2}\right)$ is equal to $j+1$ if $A_{\mathrm{Sym}(j)}^{\epsilon}\left(\Gamma_{2}\right)$ is a free $A^{0}\left(\Gamma_{2}\right)$ module for $\epsilon=0,1$. And we compute the weight of the determinant of the basis.

For $\epsilon=0,1$, let $h_{j, \epsilon}(t)$ be the Hilbert-Poincaré series of $A_{\mathrm{Sym}(j)}^{\epsilon}\left(\Gamma_{2}\right)$

$$
h_{j, \epsilon}(t)=\sum_{k \equiv \epsilon \bmod 2} \operatorname{dim}_{\mathbb{C}} A_{k, j}\left(\Gamma_{2}\right) t^{k}
$$

We put

$$
f_{j, \epsilon}(t)=\left(1-t^{4}\right)\left(1-t^{6}\right)\left(1-t^{10}\right)\left(1-t^{12}\right) h_{j, \epsilon}(t) .
$$

Then by the Hilbert-Serre theorem, we have $f_{j, \epsilon}(t) \in \mathbb{Z}[t]$.

We can compute the values of $f_{j, \epsilon}$ and its differential $f_{j, \epsilon}^{\prime}$ at $t=1$.

Proposition 3.1. For $j \in 2 \mathbb{Z}_{\geqslant 0}$, we have

$$
f_{j, \epsilon}(1)=j+1, \quad f_{j, \epsilon}^{\prime}(1)+1 / 2 j(j+1)=35 \times \begin{cases}j / 2 & \text { if } \epsilon=0 \\ j / 2+1 & \text { if } \epsilon=1\end{cases}
$$

Proof. For $k, j \in \mathbb{Z}_{\geqslant 0}$, we put $a_{k, j}=0$ if $j$ is odd and let $a_{k, 2}$ be the value given by Tsushima [13, Theorem 4]. Then we have $a_{k, j}=\operatorname{dim}_{\mathbb{C}} S_{k, j}\left(\Gamma_{2}\right)$ if $k \geqslant 5$. Here $S_{k, j}\left(\Gamma_{2}\right)$ is the cuspidal 
subspace of $A_{k, j}\left(\Gamma_{2}\right)$. By [13, Theorem 4], there exists a polynomial $g(t, s) \in \mathbb{C}[t, s]$ such that

$$
\sum_{k, j=0}^{\infty} a_{k, j} t^{k} s^{j}=\frac{g(t, s)}{q(t) r(s)}
$$

Here $q(t)$ and $r(s)$ are defined by

$$
\begin{aligned}
& q(t)=\left(1-t^{4}\right)\left(1-t^{6}\right)\left(1-t^{10}\right)\left(1-t^{12}\right), \\
& r(s)=\left(1-s^{6}\right)\left(1-s^{8}\right)\left(1-s^{10}\right)\left(1-s^{12}\right) .
\end{aligned}
$$

The polynomial $g(t, s)$ is of degree 31 with respect to $t$, of degree 34 with respect to $s$ and has 354 terms. Let $g(t, s)=\sum_{i, j} c_{i j} t^{i} s^{j}$ with $c_{i j} \in \mathbb{Z}$. For $\epsilon=0,1$, we define a polynomial $g_{\epsilon}(t, s)$ by $g_{\epsilon}(t, s)=\sum_{i \equiv \epsilon \bmod 2} c_{i j} t^{i} s^{j}$. Then $g_{\epsilon}(t, s)$ satisfies the equations

$$
\begin{gathered}
\frac{g_{\epsilon}(1, s)}{r(s)}=\frac{1+s^{2}}{\left(1-s^{2}\right)^{2}}=\sum_{j \in 2 \mathbb{Z} \geqslant 0}(j+1) s^{j}, \quad \text { for } \epsilon=0,1, \\
\frac{\partial g_{\epsilon}}{\partial t}(1, s) / r(s)+\sum_{j \in 2 \mathbb{Z} \geqslant 0} 1 / 2 j(j+1) s^{j}=\frac{35 s^{2(1-\epsilon)}}{\left(1-s^{2}\right)^{2}} \\
=35 \times \begin{cases}\sum_{j \in 2 \mathbb{Z}_{\geqslant 0}} j / 2 s^{j} & \text { if } \epsilon=0, \\
\sum_{j \in 2 \mathbb{Z}_{\geqslant 0}}(j / 2+1) s^{j} & \text { if } \epsilon=1 .\end{cases}
\end{gathered}
$$

Suppose $k>4$ is even. Then by Klingen [10] and Arakawa [2], we have

$$
A_{k, j}\left(\Gamma_{2}\right) \cong \begin{cases}\mathbb{C} \phi_{k} \oplus S_{k, 0}\left(\Gamma_{2}\right) \oplus S_{k}\left(\Gamma_{1}\right) & \text { if } j=0 \\ S_{k, j}\left(\Gamma_{2}\right) \oplus S_{k+j}\left(\Gamma_{1}\right) & \text { if } j>0 .\end{cases}
$$

Here $S_{k+j}\left(\Gamma_{1}\right)$ is the space of elliptic cusp forms of level 1 and we put $\phi_{k}=0$ if $k$ is odd. Since the denominator of the Hilbert-Poincare series of $\left\{S_{k+j}\left(\Gamma_{1}\right)\right\}_{k}$ is equal to $\left(1-t^{4}\right)\left(1-t^{6}\right)$, we have

$$
\frac{g_{\epsilon}(t, s)}{q(t) r(s)}=\sum_{j=0}^{\infty}\left(\frac{f_{j, \epsilon}(t)}{q(t)}+\frac{P_{j}(t)}{\left(1-t^{4}\right)\left(1-t^{6}\right)}+\frac{Q_{j}(t)}{\left(1-t^{2}\right)}\right) s^{j},
$$

where $P_{j}(t)$ and $Q_{j}(t)$ are polynomials. Therefore by equations (3.1) and (3.2), we obtain the assertion of the proposition.

By Hilbert's syzygy theorem, we have the following corollary (see [5, Exercise 19.14]).

Corollary 3.2. Assume $A_{\mathrm{Sym}(j)}^{\epsilon}\left(\Gamma_{2}\right)$ is a finitely generated $A^{0}\left(\Gamma_{2}\right)$-module. Put $K=$ Frac $A^{0}\left(\Gamma_{2}\right)$. Then the following statements hold:

(i) $\operatorname{dim}_{K} A_{\operatorname{Sym}(j)}^{\epsilon}\left(\Gamma_{2}\right) \otimes K=j+1$ for $\epsilon=0,1$;

(ii) if $A_{\operatorname{Sym}(j)}^{\epsilon}\left(\Gamma_{2}\right)$ is free and $\left\{f_{1}, \ldots, f_{j+1}\right\}$ is its basis then $\operatorname{det}\left(f_{1}, \ldots, f_{j+1}\right) \in A_{35 n}\left(\Gamma_{2}\right)$, where

$$
n= \begin{cases}j / 2 & \text { if } \epsilon=0 \\ j / 2+1 & \text { if } \epsilon=1 .\end{cases}
$$


4. The structure of the modules in the case of $\operatorname{Sym}(4)$ and $\operatorname{Sym}(6)$

We recall the results of Ibukiyama [7] and van Dorp [14] on the structure of the modules of $A_{\mathrm{Sym}(j)}^{\epsilon}\left(\Gamma_{2}\right)(j=4,6, \epsilon=0,1)$.

\subsection{General theory of Rankin-Cohen differential operators}

For the construction of vector-valued Siegel modular forms, Ibukiyama used Rankin-Cohen type differential operators. In this subsection, we recall the theory of Rankin-Cohen type differential operators in the degree two case.

Let $t$ be a positive integer and $r_{11}^{(i)}, r_{12}^{(i)}, r_{22}^{(i)}$ variables for $1 \leqslant i \leqslant t$. We put $R_{i}=\left(\begin{array}{ll}r_{11}^{(i)} & r_{12}^{(i)} \\ r_{12}^{(i)} & r_{22}^{(i)}\end{array}\right)$. Let $\mathbb{H}_{2}^{(1)}, \ldots, \mathbb{H}_{2}^{(t)}$ be $t$ copies of $\mathbb{H}_{2}$ and put $Z_{i}=\left(\begin{array}{cc}z_{11}^{(i)} & z_{12}^{(i)} \\ z_{12}^{(i)} & z_{22}^{(i)}\end{array}\right) \in \mathbb{H}_{2}^{(i)}$. Let $Q\left(R_{1}, \ldots, R_{t}, u\right)$ be a $\mathbb{C}$-coefficient polynomial of the components of $R_{i}$ and $u$ such that $Q$ is homogeneous of degree $j$ in $u$. For such $Q$ and holomorphic functions $f_{i}\left(Z_{i}\right)$ on $\mathbb{H}_{2}^{(i)}(1 \leqslant i \leqslant t)$, we define a function on $\mathbb{H}_{2}$ by

$$
\left\{f_{1}, \ldots, f_{t}\right\}_{Q}(Z)=\left.\left(Q\left(\partial_{Z_{1}}, \ldots, \partial_{Z_{t}}, u\right)\left(\prod_{i=1}^{t} F_{i}\left(Z_{i}\right)\right)\right)\right|_{Z_{i}=Z}
$$

Here

$$
\partial_{Z}=\left(\frac{1+\delta_{i j}}{2(2 \pi i)} \frac{\partial}{\partial z_{i j}}\right)
$$

and $\left.\right|_{Z_{i}=Z}$ means substituting $Z_{i}$ by $Z$ for all $1 \leqslant i \leqslant t$.

Theorem 4.1 (Ibukiyama [6]). Fix $k_{1}, \ldots, k_{t} \in \mathbb{Z}_{\geqslant 1}$ and $a \in \mathbb{Z}_{\geqslant 0}$. Then $Q$ satisfies

$$
\left\{\left.f_{1}\right|_{k_{1}}[g], \ldots,\left.f_{t}\right|_{k_{t}}[g]\right\}_{Q}=\left.\left\{f_{1}, \ldots, f_{t}\right\}_{Q}\right|_{k_{1}+\ldots+k_{t}+a, j}[g]
$$

for any holomorphic functions $f_{i}(1 \leqslant i \leqslant t)$ and for any $g \in \mathrm{Sp}_{2}(\mathbb{R})$ if and only if $Q$ satisfies the following two conditions.

(i) For all $A \in \mathrm{GL}_{2}, Q$ satisfies

$$
Q\left(A R_{1}{ }^{t} A, \ldots, A R_{t}{ }^{t} A, u\right)=(\operatorname{det} A)^{a} Q\left(R_{1}, \ldots, R_{t}, u A\right) .
$$

(ii) Let $X_{i}$ (for $1 \leqslant i \leqslant t$ ) be $2 \times 2 k_{i}$ matrix variables. Then $Q\left(X_{1}{ }^{t} X_{1}, \ldots, X_{t}{ }^{t} X_{t}\right)$ is pluriharmonic with respect to $X=\left(x_{i j}\right)$, that is

$$
\sum_{\nu=1}^{2\left(k_{1}+\ldots+k_{r}\right)} \frac{\partial^{2} Q}{\partial x_{i \nu} \partial x_{j \nu}}=0, \quad \text { for } 1 \leqslant i, j \leqslant 2 .
$$

We shall give examples for $Q$ satisfying the conditions (i) and (ii) in Theorem 4.1.

\subsection{Examples in the case of $t=2$}

Let $t=2$. We write $R=R_{1}=\left(r_{i j}\right), S=R_{2}=\left(s_{i j}\right), k_{1}=k$ and $k_{2}=l$. Eholzer and Ibukiyama [4] gave polynomials satisfying the conditions (i) and (ii) in Theorem 4.1 for $a=0$ and $a=2$. We introduce these polynomials.

For $k, l, m \in \mathbb{Z}_{\geqslant 0}$, we put

$$
G_{k, l, m}(x, y)=\sum_{i=0}^{m}(-1)^{i}\left(\begin{array}{c}
m+l-1 \\
i
\end{array}\right)\left(\begin{array}{c}
m+k-1 \\
m-i
\end{array}\right) x^{i} y^{m-i} .
$$


For an even $j$, we put $Q_{k, l, j}(R, S)=G_{k, l, j / 2}(r, s)$, where $r=r_{11} u_{1}^{2}+2 r_{12} u_{1} u_{2}+r_{22} u_{2}^{2}$ and $s=s_{11} u_{1}^{2}+2 s_{12} u_{1} u_{2}+s_{22} u_{2}^{2}$. Then the polynomial $Q_{k, l, j}$ satisfies the conditions (i) and (ii) in Theorem 4.1 for $a=0$. For $F \in A_{k}\left(\Gamma_{2}\right)$ and $G \in A_{l}\left(\Gamma_{2}\right)$, we put

$$
\{F, G\}_{\operatorname{Sym}(j)}=\{F, G\}_{Q_{k, l, j}} .
$$

Then we have $\{F, G\}_{\mathrm{Sym}(j)} \in A_{k+l, j}\left(\Gamma_{2}\right)$. If $j=2$, this differential operator was defined by Satoh [11].

We define a polynomial $Q_{k, l,(2, j)}(R, S, u)$ by

$$
\begin{aligned}
& 4^{-1} G_{2}(R, S) G_{k+1, l+1, j / 2}(r, s)+2^{-1}((2 l-1) \operatorname{det}(R) s-(2 k-1) \operatorname{det}(S) r) \\
& \quad \times\left(\frac{\partial G_{k+1, l+1, j / 2}}{\partial x}(r, s)-\frac{\partial G_{k+1, l+1, j / 2}}{\partial y}(r, s)\right) .
\end{aligned}
$$

Here $G_{2}(R, S)$ is defined by

$$
\begin{aligned}
G_{2}(R, S)= & (2 k-1)(2 l-1) \operatorname{det}(R+S)-(2 k-1)(2 k+2 l-1) \operatorname{det}(S) \\
& -(2 l-1)(2 k+2 l-1) \operatorname{det}(R) .
\end{aligned}
$$

Then the polynomial $Q_{k, l,(2, j)}$ satisfies the conditions (i) and (ii) in Theorem 4.1 for $a=2$. For $F \in A_{k}\left(\Gamma_{2}\right)$ and $G \in A_{l}\left(\Gamma_{2}\right)$, we put

$$
\{F, G\}_{\operatorname{det}^{2} \operatorname{Sym}(j)}=\{F, G\}_{Q_{k, l,(2, j)}} .
$$

Then we have $\{F, G\}_{\operatorname{det}^{2} \operatorname{Sym}(j)} \in A_{k+l+2, j}\left(\Gamma_{2}\right)$.

4.3. Example in the case of $t=3$ and $j=4$

Let $t=3$ and $j=4$. We put $R=\left(r_{i j}\right)_{1 \leqslant i, j \leqslant 2}, S=\left(s_{i j}\right)_{1 \leqslant i, j \leqslant 2}$ and $T=\left(t_{i j}\right)_{1 \leqslant i, j \leqslant 2}$.

We define $Q_{\operatorname{det} \operatorname{Sym}(4)}(R, S, T, u)=\sum_{i=0}^{4} Q_{i}(R, S, T) u_{1}^{4-i} u_{2}^{i}$. Here $Q_{0}(R, S, T)$ is defined by

$$
Q_{0}(R, S, T)=\left(k_{2}+1\right)\left|\begin{array}{ccc}
\left(k_{1}+1\right) r_{11} & k_{2} & k_{3} \\
r_{11}^{2} & s_{11} & t_{11} \\
r_{11} r_{12} & s_{12} & t_{12}
\end{array}\right|-\left(k_{1}+1\right)\left|\begin{array}{ccc}
k_{1} & \left(k_{2}+1\right) s_{11} & k_{3} \\
r_{11} & s_{11}^{2} & t_{11} \\
r_{12} & s_{11} s_{12} & t_{12}
\end{array}\right| .
$$

We omit the definition of $Q_{i}(R, S, T)$ if $i>0$. The polynomial $Q_{\operatorname{det} \operatorname{Sym}(4)}$ was given by Ibukiyama [7] and satisfies conditions (i) and (ii) in Theorem 4.1 for $a=1$ and $j=4$. For $f_{i} \in A_{k_{i}}\left(\Gamma_{2}\right)(i=1,2,3)$, we put

$$
\left\{f_{1}, f_{2}, f_{3}\right\}_{\operatorname{det} \operatorname{Sym}(4)}=\left\{f_{1}, f_{2}, f_{3}\right\}_{Q_{\operatorname{det} \operatorname{Sym}(4)}} .
$$

Then we have $\left\{f_{1}, f_{2}, f_{3}\right\}_{Q_{\text {det Sym }(4)}} \in A_{k_{1}+k_{2}+k_{3}+1,4}\left(\Gamma_{2}\right)$.

4.4. The structure of $A_{\mathrm{Sym}(4)}^{0}\left(\Gamma_{2}\right), A_{\mathrm{Sym}(4)}^{1}\left(\Gamma_{2}\right)$ and $A_{\mathrm{Sym}(6)}^{0}\left(\Gamma_{2}\right)$

In this subsection, we recall the result of Ibukiyama [7].

THEOREM 4.2 (Ibukiyama).

(1) The module $A_{\mathrm{Sym}(4)}^{0}\left(\Gamma_{2}\right)$ is free over $A^{0}\left(\Gamma_{2}\right)$ of rank 5 and is generated by the elements

$$
\begin{aligned}
& \left\{\phi_{4}, \phi_{4}\right\}_{\operatorname{Sym}(4)},\left\{\phi_{4}, \phi_{6}\right\}_{\operatorname{Sym}(4)},\left\{\phi_{4}, \phi_{6}\right\}_{\operatorname{det}^{2} \operatorname{Sym}(4)}, \\
& \left\{\phi_{4}, \chi_{10}\right\}_{\operatorname{Sym}(4)},\left\{\phi_{6}, \chi_{10}\right\}_{\operatorname{Sym}(4)} .
\end{aligned}
$$


(2) The module $A_{\mathrm{Sym}(4)}^{1}\left(\Gamma_{2}\right)$ is free over $A^{0}\left(\Gamma_{2}\right)$ of rank 5 and is generated by the elements

$$
\begin{aligned}
& \left\{\phi_{4}, \phi_{4}, \phi_{6}\right\}_{\operatorname{det} \operatorname{Sym}(4)},\left\{\phi_{4}, \phi_{6}, \phi_{6}\right\}_{\operatorname{det} \operatorname{Sym}(4)},\left\{\phi_{4}, \phi_{4}, \chi_{10}\right\}_{\operatorname{det} \operatorname{Sym}(4)}, \\
& \left\{\phi_{4}, \phi_{4}, \chi_{12}\right\}_{\operatorname{det} \operatorname{Sym}(4)},\left\{\phi_{4}, \phi_{6}, \phi_{12}\right\}_{\operatorname{det} \operatorname{Sym}(4)} .
\end{aligned}
$$

(3) The module $A_{\mathrm{Sym}(6)}^{0}\left(\Gamma_{2}\right)$ is free over $A^{0}\left(\Gamma_{2}\right)$ of rank 7 and is generated by the elements

$$
\begin{aligned}
& E_{6,6}, X_{8,6}, X_{10,6},\left\{\phi_{4}, \phi_{6}\right\}_{\operatorname{det}^{2} \operatorname{Sym}(6)},\left\{\phi_{4}, \chi_{10}\right\}_{\operatorname{Sym}(6)}, \\
& \left\{\phi_{4}, \chi_{12}\right\}_{\operatorname{Sym}(6)},\left\{\phi_{6}, \chi_{12}\right\}_{\operatorname{Sym}(6)} .
\end{aligned}
$$

Here $E_{6,6} \in A_{6,6}\left(\Gamma_{2}\right)$ is the Klingen-Eisenstein series associated with the Ramanujan Delta function $\Delta$, which Arakawa [2] defined in the general case. The modular forms $X_{8,6} \in A_{8,6}\left(\Gamma_{2}\right)$ and $X_{10,6} \in A_{10,6}\left(\Gamma_{2}\right)$ are theta series defined in $[\mathbf{7}, \S 6]$.

REMARK 4.3. Generators with small determinant weights often cannot be constructed by differential operators.

\subsection{The structure of $A_{\mathrm{Sym}(6)}^{1}\left(\Gamma_{2}\right)$}

We briefly recall the result of van Dorp [14].

As mentioned in the introduction, van Dorp [14] proved the module $A_{\mathrm{Sym}(6)}^{0}\left(\Gamma_{2}\right)$ is free of rank 7 and gave generators explicitly. He made a recipe for constructing a polynomial satisfying the conditions (i) and (ii) in Theorem 4.1 for $t=3, a=1$ and for all even $j \geqslant 2$. And he constructed polynomials satisfying the conditions (i) and (ii) in Theorem 4.1 for $t=3, j=6$ and $a=1$.

He also constructed a Rankin-Cohen differential operator on vector-valued Siegel modular forms, that is, for $f \in A_{k, j}\left(\Gamma_{2}\right)$ and $g \in A_{k}\left(\Gamma_{2}\right)$, he defined a Rankin-Cohen differential operator $\{f, g\} \in A_{k+l+1, j}\left(\Gamma_{2}\right)$.

He constructed five of the seven generators by Rankin-Cohen type differential operators (on scalar-valued Siegel modular forms) and the remaining two generators by $\left\{E_{6,6}, \phi_{4}\right\}$ and $\left\{X_{8,6}, \phi_{4}\right\}$. Here modular forms $E_{6,6}$ and $X_{8,6}$ are given in Theorem 4.2.

\section{Proof of the main result}

We prove our main result by numerical computation. We use Sage [12] and a Sage package for Siegel modular forms of degree two written by the author. With this package, we can compute generators $\phi_{4}, \phi_{6}, \chi_{10}, \chi_{12}$ and $\chi_{35}$ of the ring of scalar-valued Siegel modular forms and a basis of the space of scalar-valued Siegel modular forms $A_{k}\left(\Gamma_{2}\right)$. We can also compute the action of Hecke operators on vector valued Siegel modular forms and the differential operators introduced in $\S 4$ from given Siegel modular forms.

Put $V=A_{\operatorname{Sym}(j)}^{\epsilon}\left(\Gamma_{2}\right)$. By Ibukiyama [7], van Dorp [14] and Kiyuna [9], if $j(V)=4,6$ or 8, then $V$ is free of rank $j(V)+1$ over $A^{0}\left(\Gamma_{2}\right)$. Therefore by Corollary 3.2 if $f_{1}, \ldots, f_{j(V)+1}$ is its basis, then $\operatorname{det}\left(f_{1}, \ldots, f_{j(V)+1}\right) \in A_{k(V)}\left(\Gamma_{2}\right)$, where $k(V)=35(j(V) / 2+\epsilon(V))$. We define an element $\operatorname{det}(V)$ of $A_{k(V)}\left(\Gamma_{2}\right) / \mathbb{C}^{\times}$by

$$
\operatorname{det}(V)=\left[\operatorname{det}\left(f_{1}, \ldots, f_{j(V)+1}\right)\right] .
$$

Here $\mathbb{C}^{\times}$acts on $A_{k(V)}\left(\Gamma_{2}\right)$ by ordinary multiplication and $\left[\operatorname{det}\left(f_{1}, \ldots, f_{j(V)+1}\right)\right]$ is the class represented by $\operatorname{det}\left(f_{1}, \ldots, f_{j(V)+1}\right)$.

In order to prove $\operatorname{det}(V)=\left[\chi_{35}^{j(V) / 2+\epsilon(V)}\right]$, it is enough to calculate a finite number of Fourier coefficients. 
Lemma 5.1. Let $k$ be a non-negative integer and $f \in A_{k}\left(\Gamma_{2}\right)$ a scalar-valued Siegel modular form. For $(n, r, m) \in \mathbb{Z}^{3}$ with $n, m, 4 n m-r^{2} \geqslant 0$, we denote by $a((n, r, m), f)$ the $(n, r, m)$ th Fourier coefficient

$$
f(Z)=\sum_{(n, r, m)} a((n, r, m), f) \mathbf{e}\left(n z_{11}+r z_{12}+m z_{22}\right) .
$$

Here $Z=\left(\begin{array}{cc}z_{11} & z_{12} \\ z_{12} & z_{22}\end{array}\right) \in \mathbb{H}_{2}$ and $\mathbf{e}(z)=\exp (2 \pi i z)$. Assume that $a((n, r, m), f)=0$ if $n \leqslant[k / 10]$ and $m \leqslant[k / 10]$, where $[x]$ is the Gauss symbol. Then we have $f=0$.

Proof. Igusa [8] proves that $A_{k}\left(\Gamma_{2}\right)$ has a basis of forms with integral coefficients. Therefore we may assume $a((n, r, m), f) \in \mathbb{Z}$ for all $(n, r, m)$. Applying the Sturm type theorem [3] for all primes $p \geqslant 5$, we obtain the assertion of the lemma.

\subsection{Proof in the case of $A_{\mathrm{Sym}(4)}^{0}\left(\Gamma_{2}\right)$ and $A_{\mathrm{Sym}(4)}^{1}\left(\Gamma_{2}\right)$}

Since the proof is the same, we consider only the case of $A_{\mathrm{Sym}(4)}^{0}\left(\Gamma_{2}\right)$. By Lemma 5.1, we need to compute the Fourier coefficients of a representative of $\operatorname{det}\left(A_{\mathrm{Sym}(4)}\left(\Gamma_{2}\right)\right)$ and $\chi_{35}^{2}$ at $(n, r, m)$ for $0 \leqslant n, m \leqslant 7$ and $r^{2}-4 n m \leqslant 0$. For the computation of $\chi_{35}$, we use the result of Aoki and Ibukiyama [1], which we mentioned in $\S 1$. We can easily compute the other generators $\phi_{4}, \phi_{6}, \chi_{10}$ and $\chi_{12}$ because they are Siegel-Eisenstein series or written as polynomials of Siegel-Eisenstein series. The Fourier coefficients of $\left\{F_{1}, \ldots, F_{r}\right\}_{Q}$ can be written by a polynomial of the Fourier coefficients of $F_{1}, \ldots, F_{r}$ and the polynomial is determined by $Q$. Here we use the notation in $\S 4$. Thus we can confirm our statement in this case if we use the package. The following code checks Theorem 1.2 in the case when $V=A_{\operatorname{Sym}(4)}^{0}\left(\Gamma_{2}\right)$.

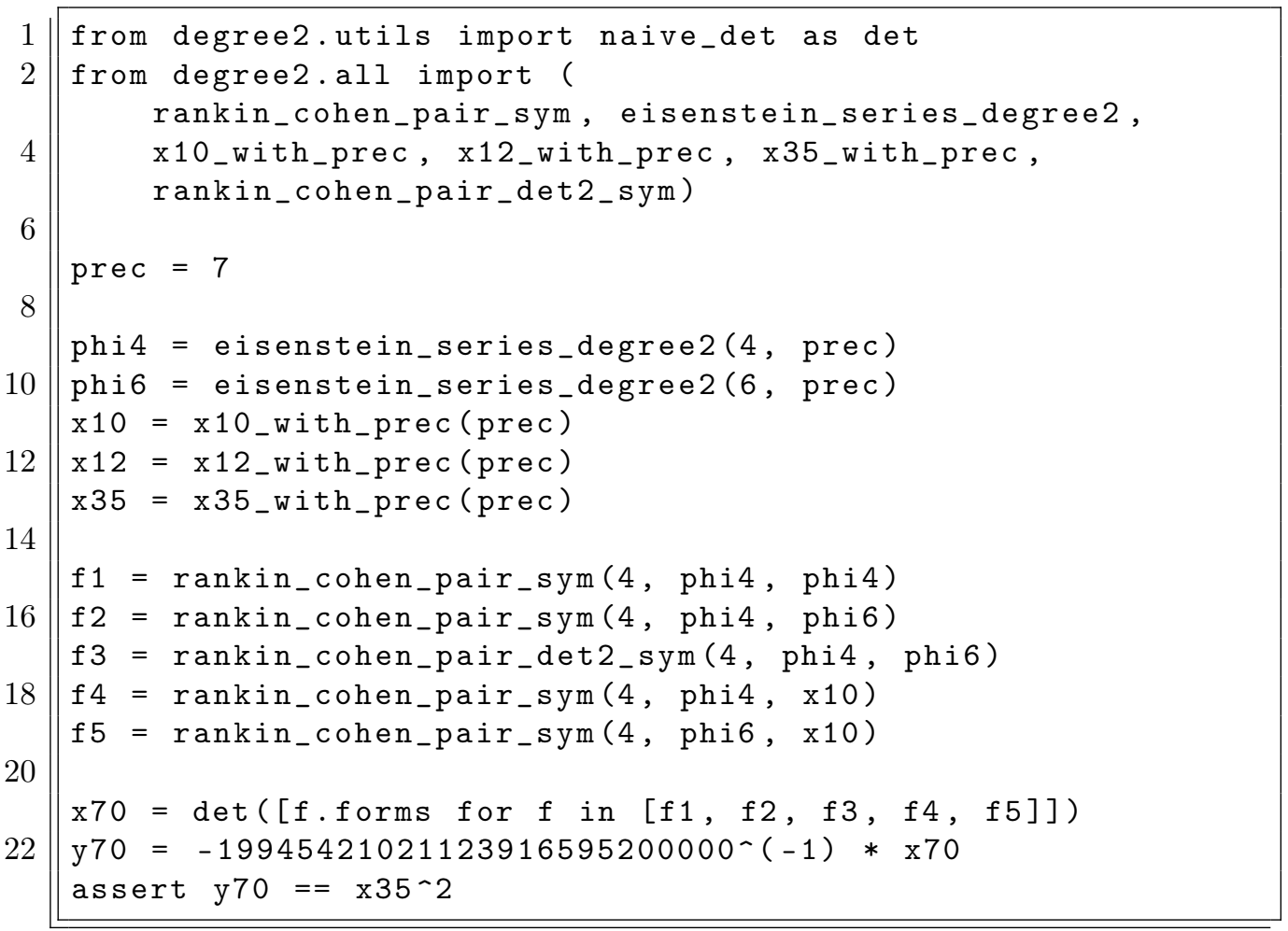


We explain the code above. In the first five lines, we load the functions used in this computation. In the seventh line, we define the variable prec, whose value is 7 . This means we compute the Fourier coefficients of Siegel modular forms for $(n, r, m)$ with $n, m \leqslant 7$. From the ninth line to the thirteenth line, we calculate $\phi_{4}, \phi_{6}, \chi_{10}, \chi_{12}$ and $\chi_{35}$. From the fifteenth line to the nineteenth line, we calculate the bases given in Theorem 4.2 and denote them by $f_{1}, \ldots, f_{5}$ respectively. In the 21 st line, we compute $\operatorname{det}\left(f_{1}, \ldots, f_{5}\right)$ and denote by $x_{70}$ the determinant. In the $22 \mathrm{nd}$ line, we calculate $-19945421021123916595200000^{-1} x_{70}$ and denote it by $y_{70}$. In the last line, we check whether $y_{70}$ is equal to $\chi_{35}^{2}$.

We can run this code if we install Sage [12] and the package 'degree2'. Running this code returns nothing. It means the main result for $V=A_{\mathrm{Sym}(4)}^{0}\left(\Gamma_{2}\right)$ is true. If the main result for this case were false, then the last line of the code would cause an error.

\subsection{Proof in the case of $A_{\mathrm{Sym}(6)}^{0}\left(\Gamma_{2}\right)$ and $A_{\mathrm{Sym}(6)}^{1}\left(\Gamma_{2}\right)$}

Since the proof is the same, we consider only the case of $A_{\mathrm{Sym}(6)}^{0}\left(\Gamma_{2}\right)$. Let $E_{6,6}$ and $X_{8,6}$ be the vector-valued Klingen-Eisenstein series and the theta series that appeared in Theorem 4.2. Since the computation of theta series is slow and we do not know the Fourier coefficients of vector-valued Klingen-Eisenstein series explicitly, we compute $\phi_{6} E_{6,6}$ and $\phi_{4} X_{8,6}$ instead. By Theorem 4.2, $\left\{\phi_{6} E_{6,6}, \phi_{4} X_{8,6}, F_{12}\right\}$ is a basis of $A_{12,6}\left(\Gamma_{2}\right)$ over $\mathbb{C}$. Here we put $F_{12}=$ $\left\{\phi_{4}, \phi_{6}\right\}_{\operatorname{det}^{2} \operatorname{Sym}(6)}$. We give another computable basis of $A_{12,6}\left(\Gamma_{2}\right)$ by $F_{12}, F_{12} \mid T(2)$ and a vector modular form constructed by a differential operator, where $T(2)$ is the Hecke operator. This method was already used by van Dorp [14].

Define $F_{k} \in A_{k, 6}\left(\Gamma_{2}\right)$ for $k=10,14,16,18$ as follows:

$$
\begin{array}{ll}
F_{10}=\left\{\phi_{4}, \phi_{6}\right\}_{\operatorname{Sym}(6)}, & F_{14}=\left\{\phi_{4}, \chi_{10}\right\}_{\operatorname{Sym}(6)} \\
F_{16}=\left\{\phi_{4}, \chi_{12}\right\}_{\operatorname{Sym}(6)}, & F_{18}=\left\{\phi_{6}, \chi_{12}\right\}_{\operatorname{Sym}(6)}
\end{array}
$$

Then by Theorem 4.2, $\left\{E_{6,6}, X_{8,6}, X_{10,6}, F_{12}, F_{14}, F_{16}, F_{18}\right\}$ is a basis of $A_{\operatorname{Sym}(6)}^{0}\left(\Gamma_{2}\right)$ over $A^{0}\left(\Gamma_{2}\right)$. We define $G_{12}, H_{12} \in A_{12,6}\left(\Gamma_{2}\right)$ by

$$
G_{12}=F_{12} \mid T(2), \quad H_{12}=\left\{\phi_{4}, \phi_{4}^{2}\right\}_{\operatorname{Sym}(8)} .
$$

Here $T(2)$ is the Hecke operator. As mentioned above, $\left\{\phi_{6} E_{6,6}, \phi_{4} X_{8,6}, F_{12}\right\}$ is a basis of $A_{12,6}\left(\Gamma_{2}\right)$ over $\mathbb{C}$. It can be checked that $\left\{F_{12}, G_{12}, H_{12}\right\}$ is also a basis. By Theorem 4.2, $\operatorname{dim}_{\mathbb{C}} A_{10,6}\left(\Gamma_{2}\right)=2$ and $\left\{\phi_{4} E_{6,6}, X_{10,6}\right\}$ is a basis. It can be checked that $\left\{\phi_{4} E_{6,6}, F_{10}\right\}$ is also a basis. Therefore we have

$$
\phi_{4} \phi_{6} \operatorname{det}\left(A_{\operatorname{Sym}(6)}^{0}\left(\Gamma_{2}\right)\right)=\left[\operatorname{det}\left(F_{10}, F_{12}, G_{12}, H_{12}, F_{14}, F_{16}, F_{18}\right)\right]
$$

in $A_{115}\left(\Gamma_{2}\right) / \mathbb{C}^{\times}$. Thus in order to prove the main result for this case, it is enough to prove that

$$
\left[\operatorname{det}\left(F_{10}, F_{12}, G_{12}, H_{12}, F_{14}, F_{16}, F_{18}\right)\right]=\left[\phi_{4} \phi_{6} \chi_{35}^{3}\right] .
$$

Since $F_{10}, F_{12}, H_{12}, F_{14}, F_{16}$ and $F_{18}$ are constructed by differential operators, we can compute these vector-valued Siegel modular forms. We can compute $G_{12}$ by $[\mathbf{2},(2.5)]$ if we compute Fourier coefficients of $F_{12}$. Since the computation of the Fourier coefficient of $G_{12}$ at $(n, r, m)$ requires the computation of the Fourier coefficient of $F_{12}$ at $(2 n, 2 r, 2 m)$, we need to compute more Fourier coefficients of $F_{12}$ than the other vector-valued Siegel modular forms. We can prove (5.1) in the same way as the case where $V=A_{\mathrm{Sym}(4)}^{0}\left(\Gamma_{2}\right)$. The source code to check (5.1) is slightly too long to show here. The source code can be found at https://github.com/ stakemori/det_vec_vald_SMFs. 
Acknowledgements. The author would like to thank the reviewers for their helpful suggestions. He also would like to thank Professor Ibukiyama for valuable comments.

\section{References}

1. H. Aoki and T. Ibukiyama, 'Simple graded rings of Siegel modular forms, differential operators and Borcherds products', Int. J. Math. 16 (2005) no. 3, 249-279.

2. T. Arakawa, 'Vector valued Siegel's modular forms of degree two and the associated Andrianov L-functions', Manuscripta Math. 44 (1983) no. 1-3, 155-185.

3. D. Choi, Y. Choie and T. Kikuta, 'Sturm type theorem for Siegel modular forms of genus 2 modulo $p$ ', Acta Arith. 158 (2013) no. 2, 129-139.

4. W. Eholzer and T. Ibukiyama, 'Rankin-Cohen type differential operators for Siegel modular forms', Int. J. Math. 9 (1998) no. 4, 443-463.

5. D. Eisenbud, Commutative algebra: with a view toward algebraic geometry, Graduate Texts in Mathematics vol. 150 (Springer, 1995)

6. T. IBukiyama, 'On differential operators on automorphic forms and invariant pluri-harmonic polynomials', Comment. Math. Univ. St. Pauli 48 (1999) no. 1, 103-118.

7. T. IвukiYama, 'Vector valued Siegel modular forms of symmetric tensor weight of small degrees', Comment. Math. Univ. St. Pauli 61 (2012) 51-75.

8. J. Igusa, 'On Siegel modular forms of genus two', Amer. J. Math. (1962) 175-200.

9. T. KIYUNA, 'Vector-valued Siegel modular forms of weight $\operatorname{det}^{k} \otimes \operatorname{Sym}(8)$ ', Preprint, 2013.

10. H. Klingen, 'Zum Darstellungssatz für Siegelsche Modulformen', Math. Z. 102 (1967) no. 1, 30-43.

11. T. SAтон, 'On certain vector valued Siegel modular forms of degree two', Math. Ann. 274 (1986) no. 2, 335-352.

12. W. A. Stein et al., Sage Mathematics Software (Version 6.1.1), The Sage Development Team, 2014, http://www.sagemath.org.

13. R. Tsushima, 'An explicit dimension formula for the spaces of generalized automorphic forms with respect to $\operatorname{Sp}(2, \mathbf{Z})$ ', Proc. Japan Acad. Ser. A Math. Sci. 59 (1983) no. 4, 139-142.

14. C. H. van Dorp, 'Generators for a module of vector-valued Siegel modular forms of degree 2', Preprint, 2013, arXiv:1301.2910.

Sho Takemori

Department of Mathematics

Kyoto University

Kitashirakawa-Oiwake-Cho

Sakyo-Ku, Kyoto, 606-8502

Japan

takemori@math.kyoto-u.ac.jp 